\title{
Hemodynamic Responses of Unfit Healthy Women at a Training Session with Nintendo Wii: A Possible Impact on the General Well-Being
}

\author{
Renato S. Monteiro-Junior ${ }^{1, *}$, Luiz F. Figueiredo ${ }^{2}$, Isabel Conceição ${ }^{2}$, Carolina Carvalho ${ }^{2}$, \\ Eduardo Lattari $^{3}$, Gioia Mura ${ }^{4}$, Sérgio Machado ${ }^{3,5}$ and Elirez B. da Silva ${ }^{6}$
}

\begin{abstract}
${ }^{I}$ Neuroscience, Federal Fluminense University (UFF); Neuroscience Laboratory of Exercise (LaNEx), Federal University of Rio de Janeiro (UFRJ), Exercise Fisiology Laboratory of Brazilian Institute of Rehabilitation Medicine (LAFExI), Rio de Janeiro, RJ, Brazil; ${ }^{2}$ Exercise Fisiology Laboratory of Brazilian Institute of Rehabilitation Medicine (LAFExI), Rio de Janeiro, RJ, Brazil; ${ }^{3}$ Laboratory of Panic and Respiration, Institute of Psichiatry, Federal University of Rio de Janeiro (IPUB/UFRJ), Rio de Janeiro, Brazil; National Institute of Translational Medicine (INCT-TM); ${ }^{4}$ Department of Public Health, Clinical and Molecular Medicine, University of Cagliari, Italy; ${ }^{5}$ Physical Activity Neuroscience, Physical Activity Sciences Postgraduate Program - Salgado de Oliveira University, Niterói, Brazil; ${ }^{6}$ Leader of Research Group of Clinical School of Physiotherapy and Professor of Master/Doctorate Program in Exercise and Sports Sciences, State University of Rio de Janeiro (UERJ)
\end{abstract}

\begin{abstract}
Aims: The purpose of this study was assess the effect of a training session with Nintendo Wii $^{\mathbb{B}}$ on the hemodynamic responses of healthy women not involved in regular physical exercise. Method: Twenty-five healthy unfit women aged $28 \pm 6$ years played for 10 minutes the game Free Run (Wii Fit Plus). The resting heart rate (RHR), systolic and diastolic blood pressures (SBP and DBP), and double (rate-pressure) product (DP) were measured before and after activity. The HR during the activity (exercise heart rate, EHR) was measured every minute. Results: A statistically significant difference was observed between the RHR $(75 \pm 9 \mathrm{bpm})$ and the mean EHR $(176 \pm 15 \mathrm{bpm})(\mathrm{P}<0.001)$. The EHR remained in the target zone for aerobic exercise until the fifth minute of activity, which coincided with the upper limit of the aerobic zone $(80 \%$ heart rate reserve $(\mathrm{HRR})+\mathrm{RHR})$ from the sixth to tenth minute. The initial $(110 \pm 8 \mathrm{mmHg})$ and final $(145 \pm$ $17 \mathrm{mmHg})$ SBP $(\mathrm{P}<0.01)$ were significantly different, as were the initial $(71 \pm 8 \mathrm{mmHg})$ and final $(79 \pm 9 \mathrm{mmHg}) \mathrm{DBP}$ $(\mathrm{P}<0.01)$. A statistically significant difference was observed between the pre- $(8.233 \pm 1.141 \mathrm{bpm}-\mathrm{mmHg})$ and postactivity $(25.590 \pm 4.117 \mathrm{bpm}-\mathrm{mmHg}) \mathrm{DP}(\mathrm{P}<0.01)$. Conclusion: Physical exercise while playing Free Run sufficed to trigger acute hemodynamic changes in healthy women who were not engaged in regular physical exercise.
\end{abstract}

Keywords: Aerobic exercise, experimental games, exergame, Nintendo Wii, physical activities, sedentary lifestyle.

\section{INTRODUCTION}

Sedentarism is one of the most influential factors for obesity $[1,2]$. The abrupt increase in body mass and the accumulation of fat in the central region of the body are potential risk factors for coronary artery disease (CAD) [2] and type II diabetes $[3,4]$. Physical exercise associated with dieting and other lifestyle changes may reduce obesity and, consequently, the risk factors for those diseases $[2,4,5]$. Although studies have shown these health benefits, much of the Brazilian population is overweight [6-9]. Therefore, innovative actions are needed to increase the demand for regular physical exercise.

Currently, new methods of physical activity are emerging. In five years of surveys on fitness trends $(2007,2008$, 2009, 2010 and 2011), physical exercise aimed at reducing body weight has maintained a low profile in the ranking of the ten most widely practiced sports (listed only in 2007 and 2011) [10], highlighting the hypothesis that overweight

*Address correspondence to this author at the Exercise Fisiology Laboratory of Brazilian Institute of Rehabilitation Medicine (LAFExI), Rua Correia Dutra, 133, Catete, Rio de Janeiro, RJ, Brazil; Tel: +55 21 2557-0001

Ramal: 1392; E-mail: renato.junior@ibmr.br people require further encouragement to become more physically active.

An innovative method that has emerged recently is virtual training with Nintendo Wii ${ }^{\circledR}$ (Kyoto, Japan), which has been proven effective in stimulating cardiovascular and metabolic responses in physically active individuals [11]. This method shows a key motivational aspect [12] and suffices to provide stimuli that generate significant physiological adaptations for physical conditioning [13]. These aspects are important for mental and physical health. However, results remain limited because such studies have often small and sometimes heterogeneous samples, which may affect the findings. Therefore, the aim of the present study was to assess the effect of a training session with Nintendo $\mathrm{Wil}^{{ }^{\mathbb{B}}}$ on the hemodynamic responses of healthy women not engaged in regular physical exercise. Our hypothesis was that intensity close to the aerobic zone could be reached.

\section{METHODS}

\section{Experimental Approach to the Problem}

Although Wii Fit Plus is a game, we hypothesized that some activities would be able to stimulate sufficiently car- 
diovascular system of sedentary individuals according international recommendations. Thus we tested sedentary women during one of these games and we verified cardiovascular variables changes.

\section{Subjects}

The evaluated study participants were 25 women aged 28 \pm 6 years who met the following inclusion criteria: a) absence of musculoskeletal injuries; b) non-engagement in a regular physical exercise program in the last six months prior to the study; and the following exclusion criteria: a) arterial hypertension; b) diabetes mellitus; and c) neurological diseases.

The volunteers were submitted to body mass and weight evaluations and were then submitted to the collection procedures. All participants briefed and freely signed the Informed Consent Form according to resolution 196/96 of the National Health Council. This study was approved by the Ethics Committee of Gama Filho University under protocol CAAE07145012.0.0000.5287, No. 132.561.

\section{PROCEDURES}

\section{All Procedures were Performed in a Single Visit to the Laboratory}

The study subjects assumed a supine position for five minutes to measure the resting heart rate (RHR). Blood pressure (BP) was then measured with the subject in a seated position. A heart rate monitor (Polar Electro FT4, Kempele Finland) and aneroid sphygmomanometer with stethoscope (Premium, Accumed Glicomed, Rio de Janeiro, Brazil) were used. The variables measured were heart rate (HR; presession and at every minute of activity), systolic blood pressure (SBP) and diastolic blood pressure (DBP) pre- and immediately post-activity. The rate pressure product $(\mathrm{DP}=\mathrm{HR}$ $x$ SBP) pre- and post-activity and mean exercise heart rate (EHR) were calculated. The mean EHR was calculated by averaging the heart rates measured in the tenth minute of play. The predicted maximum heart rate (MHR) [14] and HR reserve (HRR) were previously calculated [15].

The Free Run game of the Wii Fit Plus ${ }^{\circledR}$ bundle was used for training. Free Run requires a stationary run of the subject, which is translated in the screen in a dynamic run of his/her Mii (an avatar) in a scenario involving urban and rural landscape with the appearance of several characters along the way. During the run, a virtual trainer gives some advice to the runner in order to improve the performance. The person/gadget interaction that occurs through Wii Motion (manual remote control) includes the use of an accelerometer device, which transmits the speed of movements to the console through Bluetooth communication. Such devices allow the players to have a continuous visual feedback of their performance. This is an interesting issue, maybe to involve the mirror neurons and the proprioceptive systems, especially in unhealthy subjects or the affected by neurodegenerative disease and in populations with coordination deficits (e.g., Down, learning disabilities, ADHD, developmental coordination disorder).

A mini-trampoline was used to minimize any discomfort and impact caused by the contact of feet with the ground.
The length chosen for the activity was 10 min (minimum option of the game). The subjects were instructed to give their best performance during the exercise (with respect to bypassing the other characters). However, the pace and intensity of the race were self-selected. Wii Motion (manual control) remained in the subject's dominant hand. The subjects were asked to keep the coordinated movements of their lower and upper limbs at the same speed. The volunteers were briefed and familiarized with the movements for two minutes prior to measuring the resting heart rate.

\section{Statistical Analyses}

The SPSS 17 statistical package (SPSS Inc. South Wacker Drive, Chicago, USA) was used for the statistical data analysis. The results are shown as the mean and standard deviation. The conceptual assumptions were tested and assumed for the HR and DP data. A t-test for dependent samples was applied for the comparisons between RHR, EHR (mean HR measured at the tenth minute of activity) and DP (pre and post). The BP data did not meet the assumptions for parametric analysis and, therefore, a Wilcoxon test was applied. The significance level for all analyses was $\mathrm{P}<0.05$.

Given the difficulty of measuring the BP using the auscultatory method immediately after the activity (subject's agitation complicated the hearing of the Korotkoff sounds), the typical error of measurement (TEM) [16] was calculated to avoid a possible bias in this measurement.

\section{RESULTS}

The t-test showed a statistically significant difference between the RHR $(75 \pm 9 \mathrm{bpm})$ and mean EHR in the tenth minute of activity $(176 \pm 15 \mathrm{bpm})(\mathrm{t}=-30.084 ; \mathrm{P}<0.001)$.

The Wilcoxon test showed a statistically significant difference between the initial $(110 \pm 8 \mathrm{mmHg})$ and final $(145 \pm$ $17 \mathrm{mmHg}) \mathrm{SBP}(\mathrm{P}<0.01)$ and between the initial $(71 \pm 8$ $\mathrm{mmHg})$ and final $(79 \pm 9 \mathrm{mmHg}) \mathrm{DBP}(\mathrm{P}<0.01)$. The absolute and relative TEM of the SBP and DBP were $11 \mathrm{mmHg}$ $(9 \%)$ and $6 \mathrm{mmHg}(8 \%)$, respectively.

The paired t-test identified a difference between the pre$(8.233 \pm 1.141 \mathrm{bpm}-\mathrm{mmHg})$ and post-activity $(25.590 \pm$ $4.117 \mathrm{bpm}-\mathrm{mmHg})(\mathrm{t}=-20.69 ; \mathrm{P}<0.01) \mathrm{DP}$ values.

\section{DISCUSSION}

The present study aimed to assess the effect of a training session with Nintendo $W i i^{\mathbb{R}}$ on the hemodynamic responses of untrained healthy women. The results show a gradual increase in HR from the pre-activity period $(75 \pm 9 \mathrm{bpm})$ until the end of activity (176 $\pm 15 \mathrm{bpm}$; Fig. 1), as expected for an ongoing activity. The EHR remained in the aerobic target zone until the fifth minute of activity and coincided with the upper limit of the aerobic zone ( $80 \%$ HRR + RHR) from the sixth to the tenth minute, which is recommended for the gain and maintenance of cardiorespiratory fitness $[15,17]$. These results corroborate the findings by Noah et al. [13], who found increases of $78 \%(145.87 \pm 17.11 \mathrm{bpm}), 83 \%$ (155.12 $\pm 15.27 \mathrm{bpm})$ and $85 \%(159.41 \pm 13.91 \mathrm{bpm})$ in the predicted MHR (maximum HR) (187.80 $\pm 7.56 \mathrm{bpm})$ of twelve subjects testing a dance exergame (Dance Dance Revolution) with three different combinations. The entertainment com- 
mon to both studies enabled the aerobic effort to be well tolerated and the subjects to reach HR values in the upper limit threshold of the aerobic zone, which may be classified as moderate/strong $[15,17]$. The results of the present study show a new perspective of physical exercise towards the gain and maintenance of physical fitness of sedentary people, which may be a strategy to combat sedentarism and implement the final phase of cardiac rehabilitation.

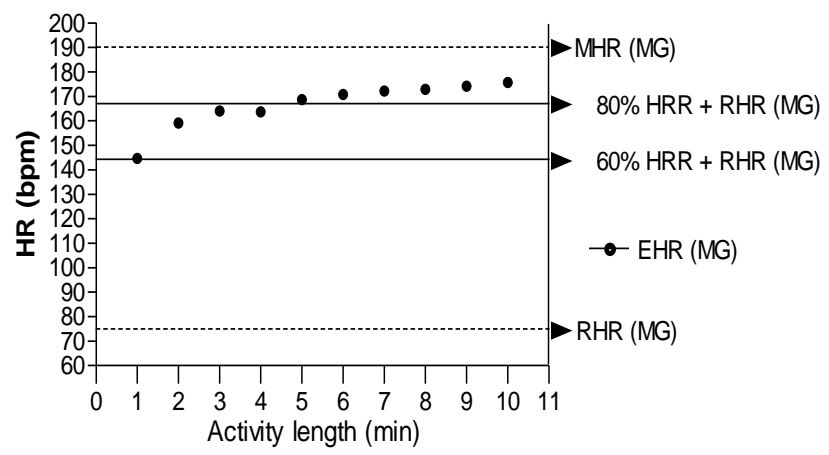

Fig. (1). Heart rate reserve (HRR) and Exercise (EHR), Resting (RHR) and Maximum (MHR) heart rates; MG - mean of group.

These results partially corroborate these by Guderian et al. [18], who found a mean exercise heart rate of $43.4 \pm$ $16.7 \%$ of the HRR when performing six Wii Fit Plus ${ }^{\circledR}$ games for 20 minutes. However, our results showed much higher values of HRR ( $>60 \%$ of the HRR + RHR) when performing Free Run for 10 minutes. This activity may be used as an alternative to increase effort in a short period of time or as a progression criterion in the prescription of aerobic training for individuals at entry or intermediate levels.

These results show that physical exercise with the Free Run game is safe because the cardiac workload remained within normal standards based on the values of SBP, DBP and DP [19]. According to the TEM of SBP and DBP (11 $\mathrm{mmHg}(9 \%)$ and $6 \mathrm{mmHg}(8 \%)$, respectively), there was only an increase in the SBP while the DBP remained stable. These results are compatible with those by Farinatti and Assis [19], who found a linear increase in the SBP and stabilization of DBP during continuous aerobic exercise.

The findings of this study support the physical activity recommendations for hypertensive patients because moderate continuous aerobic exercise is recommended for this population [20], and the activity of the sympathetic autonomic nervous system is acutely reduced after exercise [20]. However, research studies with samples from the above population must be conducted for such a conclusion.

Another key aspect was the motivation provided by the game. The level of enjoyment experienced by the participants was noticeable, albeit not measured. Graves et al. [21] showed that children, adolescents and elderly people expressed higher levels of fun during Wii Fit Plus ${ }^{\circledR}$ than during those of a common (inactive) video game or treadmill walking and jogging. However, the physiological cost of exercise was greater with the treadmill. This result was most likely due to the choice of games with very different characteristics and energy expenditure requirements (yoga, muscle conditioning, balance and aerobics). In contrast, Douris et al. [22] found a worsening sense of wellness among sedentary adults performing a 30-minute Free Run compared to a brisk walk on the treadmill. The choice of 30 minutes may have overestimated the subjects' physical capacity, leading them to a worsening sense of wellness. The cardiovascular responses during Free Run $(142.4 \pm 20.5 \mathrm{bpm})$ were higher than those during treadmill walking $(123.2 \pm 13.7 \mathrm{bpm})$. The cardiovascular response results from that study corroborate those from the present experiment, where in hemodynamic variables were stimulated enough to trigger significant acute changes in accordance with international recommendations $[10,15$, $17,20]$.

It is important to note that active lifestyle affects the psychological behavior. Depressive symptoms are related with reduced physical activity [23] and it is possible that a Wiibased physical training may have a critical role in some mood disorders. In fact there is an enjoyment perceived by different participants of this kind of intervention [21, 24] and perhaps this may increase adherence on exercise programs. Moreover physical activity reduces depressive symptoms and can be used like additional treatment in depression [25]. In addition positive changes on quality of life were found by Carta et al. [26] after exercise training protocol. Furthermore Rosenberg et al. [27] found improving on depressive symptoms, mental health-related quality of life and cognitive performance of elderly that engaged on Wii-based physical training. In this context it is possible that Wii-physical training may have similar effects with others modalities of physical exercise and with a better accessibility for people with physical disabilities because it is more feasible (home-based exercise) than physical activity in the gym. Therefore future perspectives related to "exergames" are arising and need special attention by scientific community and clinical professionals. It is noteworthy that present study is interesting but the last inferences are based on theoretical hypothesis and need be investigated.

\section{CONCLUSION}

Physical exercise performed with the active videogame Free Run of the Wii Fit Plus ${ }^{\circledR}$ sufficed to provide acute hemodynamic changes in healthy women not engaged in regular physical exercise. These changes were consistent with the recommendations of the American College of Sports Medicine for gaining and maintaining cardiorespiratory fitness.

The fact that sedentary subjects have an aversion to physical exercise highlights the need to seek strategies capable of increasing their adherence to physical activity. Physical exercise with the Free Run game was able to promote significant physiological responses and may be a key tool for healthcare professionals. The material resources used in this study are quite affordable and easy to handle. Monitoring the heart rate and blood pressure are common procedures for health professionals. Therefore, this initiative may be implemented in care clinics, gyms and clubs to attract new groups of physically active subjects.

We suggest that future longitudinal investigations be made to verify hemodynamic changes and relationship with depressive symptoms, perceived quality of life and body composition. 


\section{LIST OF ABBREVIATIONS}

$\mathrm{CAD}=$ Coronary artery disease

RHR $=$ Resting heart rate

$\mathrm{BP}=$ Blood pressure

$\mathrm{HR}=$ Heart rate

$\mathrm{SBP}=$ Systolic blood pressure

$\mathrm{DBP}=$ Diastolic blood pressure

$\mathrm{EHR}=$ Exercise heart rate

MHR $=$ Maximum heart rate

$\mathrm{HRR}=$ Heart rate reserve

TEM = Typical error of measurement

\section{CONFLICT OF INTEREST}

The authors confirm that this article content has no conflict of interest.

\section{ACKNOWLEDGEMENTS}

The authors thank Professors Monique Opuzcka and Ana Rosa Rodrigues for their help in promoting the research project and volunteer recruitment.

\section{REFERENCES}

[1] Charansonney OL, Despres J-P. Disease prevention--should we target obesity or sedentary lifestyle? Nat Rev Cardiol 2010; 7(8): 468-72.

[2] Donnelly JE, Blair SN, Jakicic JM, Manore MM, Rankin JW, Smith BK. American College of Sports Medicine Position Stand. Appropriate physical activity intervention strategies for weight loss and prevention of weight regain for adults. Med Sci Sports Exerc 2009; 41(2): 459-71.

[3] Colberg SR, Sigal RJ, Fernhall B, et al. Exercise and type 2 diabetes: the American College of Sports Medicine and the American Diabetes Association: joint position statement executive summary. Diabetes Care 2010; 33(12): 2692-6.

[4] Colberg SR, Albright AL, Blissmer BJ, et al. Exercise and type 2 diabetes: American College of Sports Medicine and the American Diabetes Association: joint position statement. Exercise and type 2 diabetes. Med Sci Sports Exerc 2010; 42(12): 2282-303.

[5] Colberg SR, Sigal RJ, Fernhall B, et al. Exercise and type 2 diabetes: the American College of Sports Medicine and the American Diabetes Association: joint position statement. Diabetes Care 2010; 33(12): e147-67.

[6] Silveira EA, Kac G, Barbosa LS. Prevalência e fatores associados à obesidade em idosos residentes em Pelotas, Rio Grande do Sul, Brasil: classificação da obesidade segundo dois pontos de corte do índice de massa corporal. Cad Saúde Pública 2009; 25: 1569-77.

[7] Buzzachera CF, Krause MP, Elsangedy HM, et al. Prevalência de sobrepeso e obesidade geral e central em mulheres idosas da cidade de Curitiba, Paraná. Rev Nutr 2008; 21: 525-33.

[8] Farias Júnior JCd, Silva KSd. Sobrepeso/obesidade em adolescentes escolares da cidade de João Pessoa - PB: prevalência e associação com fatores demográficos e socioeconômicos. Rev Bras Med Esporte 2008; 14(2): 104-8.
[9] Costa RFd, Cintra IdP, Fisberg M. Prevalência de sobrepeso e obesidade em escolares da cidade de Santos, SP. Arq Bras Endocrinol Metabol 2006; 50(1): 60-7.

[10] Thompson W. Worldwide survey of fitness trends for 2012. Health Fitness J 2011; 15(6): 9-18.

[11] Worley JR, Rogers SN, Kraemer RR. Metabolic responses to Wii Fit video games at different game levels. J Strength Cond Res 2011; 25(3): 689-93.

[12] Gil-Gomez JA, Llorens R, Alcaniz M, Colomer C. Effectiveness of a Wii balance board-based system (eBaViR) for balance rehabilitation: a pilot randomized clinical trial in patients with acquired brain injury. J Neuroeng Rehabil 2011; 8(1): 30.

[13] Noah JA, Spierer DK, Tachibana A, Bronner S. Vigorous energy expenditure with a dance exer-game. J Exerc Physiol Online. 2011; 14(4): $13-28$

[14] Tanaka H, Monahan KD, Seals DR. Age-predicted maximal heart rate revisited. J Am Coll Cardiol 2001; 37(1): 153-6.

[15] Thompson W, American College of Sports M. ACSM's resource manual for Guidelines for exercise testing and prescription. $8^{\mathrm{a}}$ ed. Wolters Kluwer Health/Lippincott Williams \& Wilkins 2009.

[16] Hopkins WG. Institute of Sport \& Recreation Research. Auckland: Sport Science; 2009 [cited 201124 de setembro]; Available from: http://sportsci.org/will/

[17] Garber CE, Blissmer B, Deschenes MR, et al. American College of Sports Medicine position stand. Quantity and quality of exercise for developing and maintaining cardiorespiratory, musculoskeletal, and neuromotor fitness in apparently healthy adults: guidance for prescribing exercise. Medicine and science in sports and exercise. 2011; 43(7): 1334-59.

[18] Guderian B, Borreson LA, Sletten LE, et al. The cardiovascular and metabolic responses to Wii Fit video game playing in middleaged and older adults. J Sports Med Phys Fitness 2010; 50(4): 43642 .

[19] Farinatti P, Assis B. Estudo da frequência cardíaca, pressão arterial e duplo-produto em exercícios contra-resistência e aeróbio contínuo. Rev Bras Ativ Fís Saúde 2000; 5(2): 5-16.

[20] Pescatello LS, Franklin BA, Fagard R, Farquhar WB, Kelley GA, Ray CA. American College of Sports Medicine position stand. Exercise and hypertension. Med Sci Sports Exerc 2004; 36(3): 533 53.

[21] Graves LE, Ridgers ND, Williams K, Stratton G, Atkinson G, Cable NT. The physiological cost and enjoyment of Wii Fit in adolescents, young adults, and older adults. J Phys Act Health 2010; 7(3): 393-401.

[22] Douris PC, McDonald B, Vespi F, Kelley NC, Herman L. Comparison between Nintendo Wii Fit aerobics and traditional aerobic exercise in sedentary young adults. J Strength Cond Res 2012; 26(4): 1052-7.

[23] Krämer LV, Helmes AW, Seelig H, Fuchs R, Bengel J. Correlates of reduced exercise behaviour in depression: the role of motivational and volitional deficits. Psychol Health 2014; 29(10): 1206-25.

[24] Franco JR, Jacobs K, Inzerillo C, Kluzik J. The effect of the Nintendo Wii Fit and exercise in improving balance and quality of life in community dwelling elders. Technol Health Care 2012; 20(2): 95-115.

[25] Pilu A, Sorba M, Hardoy MC, et al. Efficacy of physical activity in the adjunctive treatment of major depressive disorders: preliminary results. Clin Pract Epidemiol Ment Health 2007 9; 3: 8.

[26] Carta MG, Hardoy MC, Pilu A, et al. Improving physical quality of life with group physical activity in the adjunctive treatment of major depressive disorder. Clin Pract Epidemiol Ment Health 2008 26; $4: 1$.

[27] Rosenberg D, Depp CA, Vahia IV, et al. Exergames for subsyndromal depression in older adults: a pilot study of a novel intervention. Am J Geriatr Psychiatry 2010; 18(3): 221-6.

(C) Monteiro-Junior et al.; Licensee Bentham Open.

This is an open access article licensed under the terms of the Creative Commons Attribution Non-Commercial License (http://creativecommons.org/licenses/by-nc/3.0/) which permits unrestricted, non-commercial use, distribution and reproduction in any medium, provided the work is properly cited. 